\title{
Difficult Decisions
}

\section{Management in unstable angina}

\author{
D.L.H. Patterson
}

Islington and Bloomsbury Health Authorities, Whittington Hospital, St Mary's Wing, Highgate Hill, London N19 $5 N F, U K$.

That unstable angina is a serious and potentially dangerous condition which requires immediate attention is a statement with which few clinicians would disagree. However, if it is examined more closely and attempts are made to establish exactly just how serious and how dangerous, an apparent confusion emerges. Furthermore, if clinicians start to discuss the details of the immediate attention, it becomes abundantly clear that there is not only a huge choice of treatment available, but also that there is considerable debate as to the circumstances in which a particular therapy might best be used. There are several reasons for this controversy and they are worth elaborating in order to determine whether there is some order within the confusion.

Firstly, the term itself is applied to a clinical state which can be caused by a variety of pathophysiological abnormalities. ${ }^{1}$ The term was adopted to describe a condition which is neither stable angina pectoris nor myocardial infarction. It includes other diagnostic labels such as preinfarction angina, acute coronary insufficiency, intermediate coronary syndrome, crescendo angina and threatening infarction.

Secondly, many papers about the management of patients with unstable angina are based on the experience of a secondary or tertiary referral centre. They are largely concerned with patients who have failed to respond to conventional medical treatment. Confusion has been caused by extrapolating from these reported experiences to the patients presenting to a district general hospital or to their general practitioner.

Thirdly, there have been few studies that have examined the natural history of unstable angina. In those that have, it is often difficult to be certain that similar groups of patients are being described.

Finally, it is important to allude to the dangers of a high technology approach. The proximity to,

Correspondence: D.L.H. Patterson, M.D., F.R.C.P.

Received: 20 October 1987 and availability of, investigational facilities such as coronary arteriography has a considerable influence on the likelihood of the patient being investigated. The urge to do something active and invasive can become a force that is difficult for the doctor to resist. As a result a dependence on the investigation may begin to develop. Unstable angina will inevitably have a mortality and morbidity whatever the treatment used; failure is perhaps easier to accept when everything possible has been done. If the high technology approach is of demonstrable benefit, then this is good clinical practice. But what if it is not beneficial?

A better understanding of the complex pathophysiology of unstable angina may help produce some order out of the confusion. ${ }^{1}$ For many years it was believed that unstable angina was due to episodic changes in myocardial oxygen demand superimposed on rapidly advancing or completed coronary thrombosis. It is now apparent that alternative or additional mechanisms need to be considered. These include episodic reductions in the myocardial oxygen supply due to coronary artery spasm, platelet activation, thrombus formation and fibrinolysis.

\section{Natural history of unstable angina}

What information do we have about the natural history of unstable angina and how does it fit with our present understanding of the pathophysiology? Within the group of patients presenting with unstable angina some authors have attempted to identify two sub-groups which can be clinically distinguished; unfortunately there is a considerable overlap between them. One sub-group, termed by some the intermediate coronary syndrome, is characterized by recurrent angina which is prolonged, intense, unresponsive to medical treatment and associated with marked ST segment depression on the baseline electrocardiogram (ECG) or on 24 hour ST segment monitoring. This is the group that has been described as having a one year

(C) The Fellowship of Postgraduate Medicine, 1988 
mortality rate in the order of $40 \%$ and will tend to be excluded from clinical trials. The other subgroup includes patients with recent onset angina of a progressive nature, or patients with chronic angina that has changed its pattern; on the baseline ECG they tend to have minor ST segment changes only. They have a one year mortality of $2-5 \%$ and an early mortality of less than $2 \%$. Patients with progressive angina have a greater prevalence of multi-vessel disease than patients with recent onset angina. ${ }^{2,3}$

It is important to recognize extra-cardiac conditions, such as oesophageal pain, ${ }^{4}$ which can be associated with myocardial ischaemia and can mislead even the most experienced clinician. Heartburn, caused by incompetence of the lower oesophageal sphincter, produces symptoms in a similar position to that of angina. Diffuse oesophageal spasm can present with pain in this position and it may be relieved by glyceryl trinitrate and helped by calcium antagonists. Furthermore ergonovine provocation can produce oesophageal pain. ${ }^{5}$ In some patients, particularly if they have recently gained weight, chest pain of oesophageal origin can be provoked by exertion. ${ }^{4}$

Oesophageal pain and myocardial ischaemia are both common symptoms and may occur in the same patient. The co-existence of the two conditions appears too frequent to be due to chance. At an early stage in the management of the patient with unstable angina, particularly if the pain is protracted and associated with little or no ST segment change on the ECG, it is important to consider the possibility of oesophageal pain. This may be merely co-existing with the angina or it may be causing coronary artery spasm. Treatment of both conditions can result in a dramatic improvement.

\section{Management of unstable angina}

A vigorous effort must initially be undertaken to diagnose medical conditions that cause an increase in myocardial oxygen demands; these include tachyarrythmias, thyrotoxicosis, exacerbation of pre-existing heart failure and anaemia. Treatment of these problems will abolish or diminish episodes of rest pain in a proportion of patients. Although there is no proof that the cessation of cigarette smoking will improve the prognosis, it is sensible to try to persuade the patient to stop.

Evidence of stress related to 'life events' should be specifically sought. The recognition of stress and discussion of it with the patient, may be sufficient to alleviate the discomfort. In some circumstances mild sedation or removal from the emotionally taxing situation may be required.

The confidence of the physician in his/her own ability to cope with the uncertain nature and course of unstable angina is of considerable importance. If the physician only feels secure with the information gained from coronary arteriography and preferably with the patient operated upon, then this will undoubtedly be 'picked up' by the patient with a consequent worsening of the angina. A vicious cycle is thus established.

\section{Drug treatment}

A number of drugs have been used in the treatment of the patient with unstable angina. As a result of the different definitions of the condition used by authors and the problems in establishing properly designed randomized trials, it is sometimes difficult to be certain of the efficacy of a particular drug and also difficult to compare one drug with another. Furthermore it is extremely difficult to compare drug treatment with surgery or percutaneous transluminal coronary angioplasty.

\section{Nitrates}

Nitrates are a mainstay of treatment. Intravenous preparations of glyceryl trinitrate or isosorbide dinitrate are extremely effective in controlling the ischaemic episodes. The patients can be transferred to oral treatment whenever they are more stable. Despite their wide use there is little objective evidence of their efficacy.

\section{Beta-adrenoceptor blocking drugs}

There is a theoretical risk that beta-adrenoceptor blocking drugs may make unstable angina worse if the predominant mechanism relates to coronary artery spasm, but the drugs are widely used in this condition with proven benefit. Even in patients with clinical left ventricular failure at the time of their pain, the introduction of beta-adrenoceptor blocking drugs can reduce the frequency of pain and improve the heart failure. The dosage of the drug will need to be adjusted so that the resting heart rate drops to between 60 and 70 beats per minute and does not increase significantly on mild exertion. Patients who are already taking the drug at the time of development of unstable angina should be continued on it unless there are contraindications since abrupt withdrawal may cause the angina to become worse and may precipitate myocardial infarction. 


\section{Calcium channel blockers}

Calcium channel blockers represent a relatively new therapeutic approach to the treatment of unstable angina. Their place in the management is now becoming clearer. The addition of nifedipine to previous treatment with nitrate and betaadrenoceptor blockers has been shown to reduce by $50 \%$ the relative risk of failed medical therapy defined as sudden death, myocardial infarction or bypass surgery within four months. ${ }^{6}$ HINT (Holland Intervention Nifedipine-Metoprolol Trial) compared nifedipine, metoprolol and their combination with placebo in patients with unstable angina not pre-treated with a beta-adrenoceptor blocking drug, as well as the effect of nifedipine in patients with unstable angina who entered while on treatment with a beta-adrenoceptor blocking drug. ${ }^{7}$ This was a short term intervention trial and the main outcome event was recurrence of ischaemia and/or progression to myocardial infarction within 48 hours. The results are expressed as the ratios of event rates relative to placebo, and are given with $95 \%$ confidence intervals. In the groups not previously treated with beta-adrenoceptor blocking drugs, metoprolol achieved a ratio of $0.75(0.49,1.16)$; nifedipine a ratio of $1.15(0.83,1.64)$ and the combination a ratio of $0.80(0.53,1.19)$. In the group of patients already receiving a beta-adrenoceptor blocking drug the addition of nifedipine was more favourable with a ratio of $0.68(0.47,0.97)$. The slightly increased incidence of cardiac events using nifedipine alone might be explained on the basis that some patients will inevitably have already sustained a myocardial infarct; it is notoriously difficult to time the moment an infarct occurs.

\section{Aspirin}

Two major studies have shown the benefit of aspirin in reducing the likelihood of death and of myocardial infarction in patients with unstable angina. The first trial showed that $324 \mathrm{mg}$ of aspirin daily for 12 weeks significantly reduced the incidence of myocardial infarction. ${ }^{8}$ Death occurred in $3.3 \%$ of the placebo and $1.6 \%$ of the aspirin group, but the difference was not statistically significant. The mean time of starting aspirin treatment was 51 hours after admission; there is no information about whether aspirin was as effective in the group of patients with marked ST segment shifts as in the lower risk group.

The benefit of aspirin treatment is further supported in a study of the efficacy of aspirin $1300 \mathrm{mg}$ daily and sulphinpyrazone, either singly or in combination. ${ }^{9}$ The patients were followed for 2 years after presenting with unstable angina. There was no observable benefit with sulphinpyrazone. The aspirin-treated patients, when compared with placebo on an intention to treat basis, produced a statistically significant reduction of $43 \%$ in the likelihood of death or myocardial infarction. The observed benefits were similar for men and women. The aspirin was started on the eighth day after admission.

The incidence of adverse effects to aspirin appears to be dose related. A low dose of aspirin is associated with minimal or no increase in gastrointestinal or other side effects. ${ }^{10}$ If bypass surgery is performed, problems with bleeding do occur when high doses have been used; the drug will need to be stopped some days before the operation. Low dose aspirin will inhibit cyclooxygenase in platelets but not in other tissues such as the vessel wall and theoretically is preferable to using higher doses. However there is now scepticism about the clinical relevance of this precise differential dosage. ${ }^{11}$

\section{Prostaglandin $E$}

Prostaglandin E1 is an analogue of prostacyclin. It shares with it the properties of vascular smooth muscle relaxation and inhibition of platelet aggregation. In a pilot study of 19 patients with unstable angina there was a significant decrease in the number of episodes of rest angina in patients treated with intravenous infusion of prostaglandin E1. ${ }^{12}$ This eliminated the need for intravenous nitroglycerine and morphine in 10 patients. Part of these favourable influences may be related to the reduction in pre-load and after-load.

\section{Anti-coagulants}

Most of the early studies with anticoagulants were not randomized and therefore difficult to assess. ${ }^{13}$ The results of a randomized trial of heparin and atenolol versus atenolol alone, suggested that heparin, whether combined with atenolol or not, prevented infarction; atenolol alone did not. ${ }^{14}$ Unfortunately 186 of the 400 patients entered had to be withdrawn from the trial.

\section{Thrombolysis}

Streptokinase has been used with some benefit in a small group of patients with unstable angina who had not responded to conventional oral therapy. ${ }^{15}$ It has been used with good effect in vein grafts in patients with developing unstable angina after 
bypass surgery. ${ }^{16}$ Other studies in patients with unstable angina have not shown such benefit. ${ }^{17}$

Streptokinase suffers from the disadvantage that its action is not limited to dissolving fibrin; it also converts the circulating plasminogen into plasmin which will derange the plasma coagulation system. The use of fibrin-specific agents such as tissue-type plasminogen-activator (t-PA) diminishes these problems. The patient presenting with unstable angina may be ideal for this form of therapy since infarction will not have occurred in the majority; in the remainder the infarction will have just occurred. Early introduction of the thrombolytic agent is therefore possible. Further trials of this form of treatment are necessary to determine its proper place in the management.

It is important to emphasize that recanalisation of the coronary artery does not necessarily result in effective reperfusion of myocardial tissue; endothelial damage or swelling of the myocytes may obstruct the microcirculation for some time afterwards. Some undesirable sequelae may actually be caused by the reperfusion; these include arrhythmias and the accumulation of metabolites or release of free radicals which may themselves cause tissue damage. The search for suitable agents to rescue the tissues from these effects is an area of active research.

\section{Other forms of treatment}

\section{Intra-aortic balloon counter pulsation}

Patients in whom medical management has failed may be helped by intra-aortic balloon counter pulsation. ${ }^{2}$ This often results in the prompt relief of chest pain. The pain will probably recur when the counter pulsation is discontinued. This technique is therefore useful only because it allows the safe performance of coronary arteriography.

\section{Percutaneous transluminal coronary angioplasty (PTCA)}

Good results have been reported in some patients with unstable angina. Most authors have reported a higher complication rate and restenosis rate in patients with unstable angina compared with those with stable angina. The primary success rate has improved from $60 \%$ in earlier studies to $90 \%$ in more recent studies. The acute complication rate, which includes myocardial infarction, emergency bypass surgery and death is in the order of $7-12 \%$; this risk can be reduced by selecting the less seriously compromised patients. ${ }^{18}$ Initially only patients with single vessel disease were treated with
PTCA; experience is now being gained with multivessel disease but dilating only the ischaemia related vessel. ${ }^{19,20}$ Although the initial results appear as good as the single vessel group, the longer term results are less good.

De Feyter et $a .^{21}$ appear to advocate a very aggressive approach to the management of unstable angina. They performed PTCA as an emergency procedure on 60 patients with unstable angina who were refractory to treatment with maximally tolerated doses of beta-adrenoceptor blocking drugs, calcium antagonists and intravenous nitroglycerine. There were no deaths related to the procedure but $7 \%$ required emergency bypass surgery and sustained a $Q$ wave myocardial infarction. During the 6-month follow-up there was only one late death, no myocardial infarcts and 8 patients who had a recurrence of angina. It is important to emphasize that this group of patients who are refractory to adequate medical treatment comprise an extremely small proportion of the total who present with unstable angina. Nevertheless they are a group who tend to be excluded from trials. This approach should not necessarily be dismissed under the title of over enthusiasm; however, even for a tertiary referral centre, the proportion of patients who had PTCA or bypass surgery, either as an emergency or electively, seems extremely high. Only $16 \%$ of the patients were managed with medical therapy alone. It again raises the possibility that the approach of the unit can make the unstable angina worse thus justifying the subsequent therapy.

\section{Coronary artery bypass grafting}

This form of treatment has been shown to eliminate or markedly decrease anginal pain in patients with unstable angina. ${ }^{22}$ There is no clear evidence from randomized trials that surgery decreases mortality or the incidence of acute myocardial infarctions in patients with unstable angina. ${ }^{23,24}$ In the recently reported VA trial ${ }^{25}$ there were no differences in the myocardial infarction rates $(12.2 \%$ vs $11.7 \%)$ or two year survival rates $(92 \%$ vs $95 \%)$ between those treated medically and those treated surgically; the latter held true whether the results were analysed on an intention to treat basis, or the treatment actually received. Only in the subset of patients with ejection fractions between $0.30-0.59$ was there a survival advantage to the surgical group ( $86 \%$ vs $96 \%$ ). The operative mortality of patients with unstable angina varies from $0.9 \%$ to $10.3 \% .^{25,26}$ The surgical mortality rates are improving with better understanding of myocardial preservation but still must be considered in the 
risk/benefit equation. It is also important to remember the adverse effect that the female sex and age has on the risk of surgery; the former doubles the risk and the risk of surgery progressively increases from the age of 65 .

Any comparison of the mortality rates between PTCA and bypass surgery must be interpreted with great care; the surgical group will inevitably include more patients with multivessel disease and worse left ventricular function.

The criteria for early surgery are still not clear; surgery undoubtedly has a place for patients who fail to respond to adequate medical treatment or, having initially responded, deteriorate in the succeeding weeks or months. In the subgroup of patients with the intermediate coronary artery syndrome bypass surgery has been shown to reduce the mortality; 27 in contrast, the recently reported VA trial found no difference in myocardial infarction rates or death in patients who satisfied similar criteria for classifying as the intermediate coronary artery syndrome. ${ }^{25}$

\section{Laser angioplasty}

This therapeutic technique is still at a very early stage of development. Its place in the management of patients has yet to be determined.

\section{Whom to treat with what and when?}

It would be helpful to be able to identify, at the time of presentation, the patient likely to do poorly on continued medical therapy. Limited data exist to help with this clinical dilemma. The most important variable predictive of failure of medical therapy has been shown to be the percentage of left ventricular myocardium at risk of ischaemia. ${ }^{6.28}$ The addition of nifedipine was the second most powerful variable influencing success of medical therapy; it reduced by $50 \%$ the relative risk of failing medical therapy within 18 months follow-up. A history of cigarette smoking exerts a detrimental influence on the success of medical therapy. Global electrocardiographic changes during episodes of angina are also predictive of failure of medical therapy, probably because they reflect extensive coronary disease.

Twelve lead electrocardiograms recorded during episodes of chest pain have been used to identify those individuals with especially poor long term prognosis, even without angiography. ${ }^{29}$ The magnitude of such twelve lead ST segment alterations during, or immediately following chest pain, is related to the angiographic extent of underlying coronary artery disease. Since transient myocardial ischaemia may provoke ST segment alterations without associated chest discomfort, continuous ambulatory monitoring may give additional information. Nevertheless the results of ST segment shifts have to be interpreted with some caution since they can occur in people in response to a change in posture or in relation to hyperventilation. ${ }^{30,31,32}$ Transient ST segment alterations and/or ventricular tachycardia demonstrated on ambulatory monitoring can identify a high risk subgroup who have left main stem or three vessel coronary artery disease and/or impaired three month prognosis. ${ }^{33}$

Once the patient is reasonably stable, exercise testing has been used to determine the necessity of coronary arteriography and subsequent coronary artery bypass surgery or angioplasty. ${ }^{34}$ The advantage of surgical management based on this approach has not been evaluated. Exercise testing may nevertheless help separate the low risk patient for whom surgery has least to offer from the high risk patient for whom surgery may be most beneficial.

With such an array of possibilities the physician is in some difficulty knowing which is the right form of treatment to use and when to use it. The balance of evidence suggests that medical treatment has most to offer in the treatment of unstable angina; with greater understanding of the pathophysiology and the ability to influence the fibrinolytic system, there is scope for even better results. Surgery confers no benefit for the vast majority of patients. Angioplasty is a relatively new treatment and is being successfully practised by some enthusiasts; its place at the moment should be restricted to a few centres and its results properly compared with medical or surgical treatment. It is far too early for it to become an accepted form of treatment for unstable angina.

The following steps are suggested for the treatment of the patient presenting with unstable angina (Table I). It should be emphasized that the vast majority of patients will settle with the appropriate medical treatment and it is therefore unwise to progress too swiftly through the various stages unless the pain remains unresponsive to the measures instituted and there is marked and widespread ST segment depression either on the baseline ECG, with pain or during 24 hour ST segment monitoring. The risk of death is small. There is a risk of a myocardial infarct; the experience in $\mathrm{HINT}^{7}$ and the recently reported VA $^{25}$ study, demonstrate that the vast majority of infarcts occur within 6 hours of presenting with unstable angina. Only very few occur later. Treatment designed to prevent progression to 
Table I Suggested treatment plan for unstable angina

Stage 1.

Remove from taxing environment

Exclude any condition causing an increase in myocardial oxygen demand

Identify and discuss areas of stress

Use sublingual or oral nitrates as necessary

Sedate if necessary

Stage 2. If episodes of pain persist after 12-24 hours:

Start intravenous isosorbide or nitroglycerine infusion

Start a beta-adrenoceptor blocking drug

Use diamorphine for pain and/or euphoriant effect if necessary

Start aspirin and/or heparin

Monitor rhythm

Stage 3. If episodes of pain persist after 48-60 hours:

Ensure that patient is adequately beta-blocked

Start a calcium antagonist

Stage 4. If episodes of pain persist after 84-96 hours:

Consider use of thrombolytic agent

Or arrange coronary arteriography

Stage 5. If episodes of pain persist after 108-120 hours:

Arrange bypass surgery or, in certain patients, PTCA

\section{References}

1. Patterson, D.L.H. Unstable angina. Postgrad Med J 1988, 64: 196.

2. Servi, S., Ardissino, D., Collarini, L. et al. Management of crescendo angina. Eur Heart $J$ 1985, 6: F83-86.

3. Victor, M.F., Likoff, M.J., Mintz, G.S. \& Likoff, W. Unstable angina pectoris of new onset: a prospective clinical and arteriographic study of 75 patients. $\mathrm{Am} \mathrm{J}$ Cardiol 1981, 47: 228-232.

4. Schofield, P.M., Bennett, D.H., Whorwell, P.J. et al. Exertional gastro-oesophageal reflux: a mechanism for symptoms in patients with angina and normal coronary arteriograms. Br Med J 1987, 294: 14591461.

5. Dart, A.M., Davies, H.A., Lowndes, R.H. et al. Oesophageal spasm and angina: diagnostic value of ergometrine (ergonovine) provocation. Eur Heart $J$ 1980, 1: 91-95.

6. Gerstenblith, G., Onyang, P., Achuff, S. et al. Nifedipine in unstable angina: A double-blind randomised trial. $N$ Engl J Med 1982, 306: 885-889.

7. Holland Interuniversity Nifedipine/Metoprolol Trial (HINT) Research Group. Early treatment of unstable angina in the coronary care unit: a randomised, double blind, placebo controlled comparison of recurrent angina in patients with nifedipine or metoprolol or both. Br Heart J 1986, 56: 400-413. myocardial infarction will therefore have a very limited effect because it is usually instituted too late. Early treatment with a fibrinolytic agent needs to be further evaluated. The diagnosis of myocardial infarction and its separation from myocardial ischaemia can be difficult and is often made retrospectively. Moreover the infarction may actually remove the acutely ischaemic myocardium and the unstable angina may thereby 'settle'.

Once the angina has settled the patients can be assessed after some weeks or months in a similar way to those patients with stable angina. Unfortunately the management of this group of patients also remains controversial. ${ }^{35}$ Early angiography is advocated by some; if the patient is having few symptoms and has mobilised well, an exercise test may give reassuring information which will allow time to pass and the confidence of the patient and the clinician to rise. An angiogram can then be deferred until there is a return of symptoms. In most patients it usually proves possible to reduce some of the medications over the ensuing months. There is a very small group of patients who continue to have some symptoms or who do not have a very satisfactory exercise test; an early angiogram will give additional information and, together with the clinical information, enable a more informed decision to be made about bypass surgery or perhaps PTCA.

8. Lewis, D.H., Davis, J.W., Archibald, D.G. et al. Protective effects of aspirin against acute myocardial infarction and death in men with unstable angina. $N$ Engl J Med 1983, 309: 396-403.

9. Cairns, J., Gent, M., Singer, J. et al. A study of aspirin and/or sulfinpyrazone in unstable angina. Circulation 1984, 70: 415.

10. Graham, D.Y. \& Smith, J.L. Aspirin and the stomach. Ann Int Med 1986, 104: 390-398.

11. Patrono, C. Aspirin for the prevention of coronary thrombosis: current facts and perspectives. Eur Heart $J$ 1986, 7: 454-459.

12. Siegel, R.J., Shah, P.K., Nathan, M. et al. Prostaglandin E1 infusion in unstable angina: effects on anginal frequency and cardiac function. Am Heart $J$ 1984, 108: 863-868.

13. Wood, P. Acute and subacute coronary insufficiency. Br Med J 1961, 1: 1779.

14. Telford, A. \& Wilson, C. Trial of heparin versus atenolol in prevention of myocardial infarction in intermediate coronary syndrome. Lancet 1981, i: $1225-1228$.

15. Lawrence, J.R., Shepard, J.T., Bone, I. et al. Fibrinolytic therapy in unstable angina pectoris: a controlled clinical trial. Thrombosis Res 1980, 17: 767.

16. Mandelkorn, J.B., Wolf, N.M., Singh, S. et al. Intracoronary thrombus in non-transmural 
myocardial infarction and in unstable angina. Am J Cardiol 1983, 52: 1-6.

17. Rentrup, P., Blanke, H., Karsch, K.A. et al. Selective Fntracoronary thrombolysis in acute myocardial infarction and unstable angina pectoris. Circulation 1981, 63: 307-317.

18. Steffinino, G., Meier, B., Finci, L. \& Rutihauser, W. Follow up results of treatment of unstable angina by coronary angioplasty. Br Heart $J$ 1987, 57: 416-419.

19. De Feyter, P.J., Serruys, P.W., Arnold, A. et al. Coronary angioplasty of the unstable angina related vessel in patients with multivessel disease Eur Heart $J$ 1986, 7: 460-467.

20. Wohlgelernter, D., Clemans, M., Highman, H.A. \& Zaret, B.L. Percutaneous coronary angioplasty of the 'culprit lesion' for management of unstable angina pectoris in patients with multivessel coronary artery disease. Am J Cardiol 1986, 58: 460-464.

21. De Feyter, P.J., Serruys, P.W., van den Brand, M. et al. Emergency coronary angioplasty in refractory unstable angina. $N$ Engl $J$ Med 1985, 313: 342-346.

22. Rahimtoola, R.H. Coronary bypass surgery for unstable angina. Cirulcation 1984, 69: 842.

23. Russel, R.O., Rackley, C.E. \& Kouchonkos, N.T. Unstable angina pectoris: management based on available information. Circulation 1982, 65: II-72.

24. Unstable Angina Pectoris Study Group: Unstable angina pectoris national cooperative study group to compare medical and surgical therapy. II In-hospital experience and initial follow-up results in patients with one, two and three vessel disease. Am J Cardiol 1978, 42: 839-848.

25. Luchi, R.J., Scott, S.M., Denpress, R.H. and the Principal Investigators and their Associates of Veterans Administration Cooperative Study no 28. Comparison of medical and surgical treatment for unstable angina. $N$ Engl J Med 1987, 316: 977-984.

26. McCormick, J.R., Schick, E.C., McCabe, C.H. et al. Determinants of operative mortality and long term survival in patients with unstable angina: the CASS experience. J Thorac Cardiovasc Surg 1985, 89: 683688.

27. Bertolasi, C.A., Tronge, J.E., Riccitelli, P. et al. Natural history of unstable angina with medical therapy. Chest 1976, 70: 596-605.

28. Ouyang, P., Brinker, J.A., Mellits, D. et al. Variables predictive of successful medical therapy with unstable angina: selection by multivariate analysis from clinical, electrocardiographic, and angiographic evaluations. Circulation 1984, 70: 367-376.

29. Gazes, P.C., Mobley, E.M., Farris, H.M. et al. Preinfarction (unstable) angina - a prospective study. Ten year follow up. Circulation 1973, 48: 331.

30. Robson, D.J. \& Belton, S. ST-segment changes in normal men during ambulatory electrocardiography. Eur Heart J 1986, 7: 223-226.

31. Yu, P.N., Yim, B.J.M. \& Stanfield C.A. Hyperventilation syndrome. Changes in the electrocardiogram, blood gases and electrolytes during voluntary hyperventilation. Arch Int Med 1959, 103: 70.

32. Armstrong, W.F., Jordan, J.W., Morris, S.N. \& McHenry, P.L. Prevalence and magnitude of ST segment and $T$ wave abnormalities in normal men during continuous ambulatory electrocardiography. Am J Cardiol 1982, 49: 1638-1642.

33. Johnson, S.M., Mauritson, D.R., Winniford, M.D. et al. Continuous electrocardiographic monitoring in patients with unstable angina pectoris: Identification of a high-risk subgroup with severe coronary disease, variant angina, and/or impaired early prognosis. $\mathrm{Am}$ Heart $J$ 1982, 103: 4-12.

34. Nixon, J.V., Hillert, M.C., Shapiro, W. \& Smitherman, T.C. Submaximal exercise testing after unstable angina. Am Heart J 1980, 99: 772-777.

35. Patterson, D.L.H., Treasure, T., Williams, A. et al. In Patterson, D.L.H. (ed) The Management of Angina Pectoris. Castle House Publications, Tunbridge Wells, Kent, 1987. 